Conclusions In conclusion, perioperative statins use may reduce the risk of all-cause mortality of HCC patients after curative liver resection. Further prospective studies and larger randomized controlled trials (RCTs) are warranted to validate the conclusion.

\section{IDDF2020-ABS-0218 THE VALUES OF ACOUSTIC RADIATION FORCE IMPULSE ELASTOGRAPHY OF LIVER IN EVALUATING THE DEGREE OF LIVER FIBROSIS IN ALCOHOLIC LIVER DISEASE}

${ }^{1}$ Ha Phuong Linh*, ${ }^{2}$ Tran Huu Ngoc. 'Department of Internal Medicine, 103 Cam Khe Clinic, Vietnam; ' 2 Department of Internal Medicine, Hung Vuong Hospital, Vietnam

\subsection{6/gutjnl-2020-IDDF.170}

Background Prevalence of alcoholic liver disease (ALD) is increasingly elevated all over the world. The degree of liver fibrosis (LF) is an important factor that affects the treatment and prognosis. Acoustic radiation force impulse (ARFI) elastography is a noninvasive and a new way of assessing LF.

Aims To evaluate the diagnostic performance of ARFI elastography for assessing LF in ALD.

Methods This prospective study included 40 patients with ALD at Thai Nguyen National Hospital from June 2019 to February 2020. These patients had been diagnosed with ALD according to the guideline of the AASL 2010. Collecting samples for laboratory testing. Liver stiffness measurement was done using ARFI elastography. Liver biopsy was graded using Metavir classification.

Results Majority were in age group of 41-50 years (63\%). The mean ARFI elastometry showed $2.71 \pm 0.59 \mathrm{~m} / \mathrm{s}$ (range $0.86-4.53$ ) in 40 male patients. Shear wave velocity (SWV) significantly correlated with the fibrosis stage. The area under the ROC curves (AUROCs) for diagnosis of $\geq F 2$ and $\geq$ F3 were 0.87 (95\% CI: $0.78-0.95)$ and 0.89 (95\% CI: $0.81-0.97)$, respectively. The cut-off values of SWV for $\geq$ F2 and $\geq$ F3 were $1.98 \mathrm{~m} / \mathrm{s}$ (Se 78.25\%, Sp 88.37\%, PPV $85.6 \%$ and NPV $82.4 \%$ ) and $\geq 2.31 \mathrm{~m} / \mathrm{s}$ (Se 95.68\%, Sp 85.58\%, PPV 71.6\%, NPV 97.8\%), respectively. APRI significantly correlated with the fibrosis stage. AUROCs for diagnosis of $\geq$ F2 and $\geq$ F3 were 0.8 (95\% CI: $0.58-0.76)$ and 0.78 (95\% CI: $0.84-0.82$ ), respectively. The cut-off values of APRI for diagnosis of $\geq$ F2 and $\geq$ F3 were $\geq 0.62$ (Se $51.1 \%$, Sp $87.13 \%$, PPV $72.3 \%$ and NPV 68.1\%) and $\geq$ 1.171 (Se 42\%, Sp 95.52\%, PPV 81\%, NPV 83.1\%), respectively.

Conclusions Increasing SWV correlates with a higher degree of LF. ARFI elastography is a noninvasive, reliable, and repeatable diagnostic test in evaluating the degree of LF.

\section{IDDF2020-ABS-0219 NEUTROPHIL LYMPHOCYTE RATIO AND PLATELET LYMPHOCYTE RATIO ARE EXCELLENT MARKERS FOR PREDICTING SURVIVAL AND SEVERITY OF HEPATOCELLULAR CARCINOMA}

Sagar Walinjkar*, Anil Arora, Ashish Kumar, Praveen Sharma, Naresh Bansal. Institute of Liver, Gastroenterology and Pancreaticobiliary Sciences, Sir Ganga Ram Hospital, India
Background Hepatocellular carcinoma (HCC) is the 5th most common malignancy worldwide. Its clinical presentation in terms of its severity is quite variable, and survival in its advanced stages is poor. Neutrophil lymphocyte ratio (NLR) and Platelet lymphocyte ratio (PLR) are the markers which have been proven useful in prognostication of many malignancies. We aimed to evaluate the predictive value of NLR and PLR in prognosticating patients with HCC and to study its association with severity of HCC.

Methods Patients with newly diagnosed HCC getting admitted between Aug 2018 and Jan 2020 were included in the study. The imaging features, ECOG status and liver functional status were analyzed, and BCLC staging was done. Patients were given appropriate treatment according to the BCLC stage. Pretreatment NLR and PLR were calculated from differential leukocyte count. Repeat imaging was done at 1 and 3 months post-treatment. Patients were followed up for 6 months for survival. Optimum cut off values of NLR and PLR were calculated by ROC curve analysis. The OS at 6 months was compared on Kaplan Meier curve using high and low NLR, PLR values. Association between NLR and PLR was also tested with BCLC stages, recurrence-free and progression-free survival.

\section{OVERALL SURVIVAL AT 6 MONTHS}
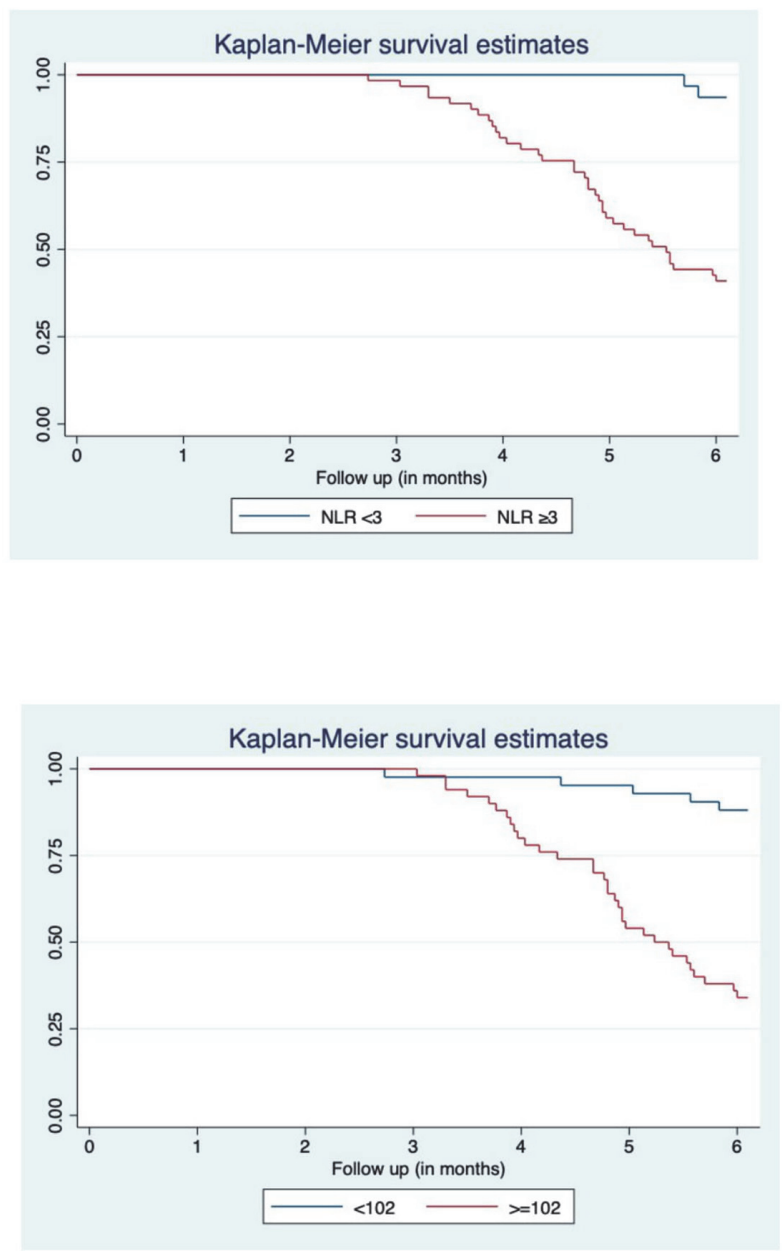

Abstract IDDF2020-ABS-0219 Figure 1 Kaplan meier curves for overall survival at 6 months according to NLR and PLR 